drugs, while two patients underwent surgery in addition to chemotherapy.

As in other reference centres, the E. Morelli Hospital needs to transfer out all admitted cases to the hospitals referring them for specialised treatment, when culture conversion and clinical stability have been achieved. Patients were transferred out after a median (IQR) hospital stay of 75.5 (51.5-127.5) days; 12 (100\%) out of 12 and nine (75\%) out of 12 achieved sputum-smear and culture conversion, after a median (IQR) time of 40.5 (24-64) and 70 (44-95) days, respectively. As of June 2011, one patient was cured, two had died and nine were still under treatment.

Four $(33.3 \%)$ cases reported adverse events, two being major (16.7\%; neuropathy and low platelet count, needing temporary interruption of linezolid) and two minor (neuropathy and mild anaemia). All adverse events were reversible.

In conclusion, despite the intrinsic difficulty of evaluating the safety and tolerability of linezolid (administered within different regimens including multiple anti-TB drugs guided by drug susceptibility testing), the study results are consistent with the findings described by VILLAR et al. [1] and SCHECTER et al. [10]. Based on the results of the study, the dose of linezolid has been reduced in Sondalo from a minimum of 450 to a maximum of $600 \mathrm{mg} \cdot$ day $^{-1}$ (determined by kinetics performed on all cases).

At present, a systematic review including information from the patients treated with linezolid globally is probably the easiest option to better define the efficacy, safety and tolerability of the drug in the treatment of MDR-/XDR-TB.

\section{S. De Lorenzo*, R. Centis", L. D’Ambrosio", G. Sotgiu" and G.B. Migliori"}

*E. Morelli Hospital, Reference Hospital for MDR and HIV TB, Sondalo, "World Health Organization Collaborating Centre for Tuberculosis and Lung Diseases, Fondazione S. Maugeri, Care and Research Institute, Tradate, and "Dept of Biomedical Sciences, University of Sassari, Sassari, Italy.

Correspondence: G.B. Migliori, World Health Organization Collaborating Centre for Tuberculosis and Lung Diseases, Fondazione S. Maugeri, Care and Research Institute, Via
Roncaccio 16, 21049, Tradate, Italy. E-mail: giovannibattista. migliori@fsm.it

Support Statement: This study was supported by the current research funds of the participating institutions. For this publication, the research leading to these results has received funding from the European Community's Seventh Framework Programme (FP7/2007-2013) under grant agreement FP7223681.

Statement of Interest: None declared.

\section{REFERENCES}

1 Villar M, Sotgiu G, D'Ambrosio L, et al. Linezolid safety, tolerability and efficacy to treat multidrug- and extensively drug-resistant tuberculosis. Eur Respir J 2011; 38: 730-733.

2 World Health Organization. Multidrug and extensively drug resistant TB (M/XDR-TB): 2010 global report on surveillance and response. Publication No. WHO/HTM/TB/2010.3. Geneva, World Health Organization, 2010.

3 Migliori GB, Besozzi G, Girardi E, et al. Clinical and operational value of the extensively drug-resistant tuberculosis definition. Eur Respir J 2007; 30: 623-626.

4 Falzon D, Jaramillo E, Schünemann HJ, et al. WHO guidelines for the programmatic management of drug-resistant tuberculosis: 2011 update. Eur Respir J 2011; 38: 516-528.

5 Sotgiu G, Ferrara G, Matteelli A, et al. Epidemiology and clinical management of XDR-TB: a systematic review by TBNET. Eur Respir J 2009; 33: 871-881.

6 Migliori GB, Eker B, Richardson MD, et al. A retrospective TBNET assessment of linezolid safety, tolerability and efficacy in multidrug-resistant tuberculosis. Eur Respir J 2009; 34: 387-393.

7 Yew WW, Lange C, Leung CC. Treatment of tuberculosis: update 2010. Eur Respir J 2011; 37: 441-462.

8 Yew WW, Chang KC, Chau CH. What is the optimal dosage of linezolid in treatment of complicated multidrug-resistant tuberculosis? Eur Respir J 2009; 34: 1492-1494.

9 Udwadia ZF, Sen T, Moharil G. Assessment of linezolid efficacy and safety in MDR- and XDR-TB: an Indian perspective. Eur Respir J 2010; 35: 936-938.

10 Schecter GF, Scott C, True L, et al. Linezolid in the treatment of multidrug-resistant tuberculosis. Clin Infect Dis 2010; 50: 49-55.

\title{
Octreotide treatment of idiopathic pulmonary fibrosis: a proof-of-concept study
}

\section{To the Editors:}

Idiopathic pulmonary fibrosis (IPF) is the most frequent form of idiopathic interstitial pneumonia. It is a chronic, progressive and fatal disease of unknown aetiology, characterised by histological features of usual interstitial pneumonia (UIP). Disease progression is marked by worsening dyspnoea, progressive loss of lung volume, abnormal gas exchange and poor quality of life. Median survival after diagnosis is $3-5$ yrs. Currently, pirfenidone is the only drug approved in Europe for the treatment in IPF, as it has been shown to slow the decline of lung function [1]. However, no effect on survival has been demonstrated until now.

Somatostatin is an endogenous cyclic peptide initially identified as a regulator of growth hormone secretion. In humans, it has been shown to bind with equal efficiency to five receptors: sst1, sst2A, sst3, sst4 and sst5. We have recently shown that the sst2A receptor is highly expressed in fibrotic lung tissue in IPF patients 
and that the uptake of octreotide, a somatostatin analogue that has a high affinity for sst2A, is elevated in the lungs of IPF patients and is correlated with the severity of lung fibrosis [2, 3]. Octreotide has been widely used for the treatment of neuroendocrine tumours for $>20$ yrs. In this study, we investigated the safety and efficacy of octreotide as a therapy for IPF.

This was an open-label, proof-of-concept, non-randomised, noncontrolled, multicentre phase II study performed in France between October 2006 and April 2008 to evaluate the safety and efficacy of intramuscular long-acting octreotide in patients with IPF. The trial was registered with ClinicalTrials.gov (identifier number NCT00463983). Patients had to be aged $\geqslant 40 \mathrm{yrs}$ and meet the diagnostic criteria for IPF described in the American Thoracic Society/European Respiratory Society consensus statement [4]. In patients aged $<50$ yrs, a lung biopsy showing a UIP pattern was required for inclusion. Inclusion criteria in patients aged $>50$ yrs required either a computed tomography (CT) scan showing "definite" IPF features or one showing "probable" features and a lung biopsy showing histological features of UIP. High-resolution CT was reviewed by two radiologists (M-P. Debray and M. Brauner) before confirming inclusion. Exclusion criteria included previous treatment with a somatostatin agonist, such as octreotide, and hypersensitivity to octreotide. Concomitant medication for IPF was not permitted during the study period and all prior IPF medication, except for prednisone ( $\leqslant 10 \mathrm{mg}$ per day), was stopped $\geqslant 6$ weeks before inclusion. Long-acting octreotide (30 mg) was administered intramuscularly every 4 weeks for 1 yr (12 injections, 48 weeks).

Recent clinical studies have shown that a decline in forced vital capacity (FVC) of $\geqslant 10 \%$ from baseline over a period of $6-$ 12 months is a strong predictor of mortality in IPF patients [5]. Hence, the primary assessment in this study was treatment failure, defined by the percentage of patients who died or whose FVC decreased by $\geqslant 10 \%$ between two consecutive measurements (with a 12-week interval) compared with baseline. These patients were termed "decliners". Secondary assessments consisted of various lung function tests, high-resolution CT, clinical evaluation, quality of life assessment (St George's Respiratory Questionnaire) and biochemical assays. These assays included measurement of serum octreotide (by a radioimmunoassay) and fibrosis biomarkers (by ELISA), namely CC chemokine ligand (CCL)2, which is a marker of progression in IPF patients, and CCL-18, surfactant protein-D and Krebs von den Lungden-6, which are associated with prognosis [6]. The safety profile was assessed based on reports of adverse events, routine physical examinations and laboratory determinations. Statistical analysis was performed using SAS version 9.1 (SAS Institute Inc., Cary, NC, USA). A linear regression method was applied to replace the missing data from patients who discontinued prematurely. This method was considered appropriate for a progressive disease such as IPF, where a continuous decrease of lung function is expected in most patients.

We enrolled and followed up 25 patients who received at least one octreotide injection. 17 patients completed the study (12 injections of $30 \mathrm{mg}$ octreotide every 4 weeks). Interestingly, only eight (32\%) patients had been diagnosed with IPF $>3$ yrs previously. Eight patients discontinued between 16 and 32 weeks because of adverse events either related or not related to octreotide treatment (one and three patients, respectively), or worsening of IPF symptoms (four patients) as indicated by a decrease in FVC or in diffusing capacity of the lung for carbon monoxide $(D \mathrm{~L}, \mathrm{CO})$ of $>10 \%$ from the baseline.

Among the 17 patients who completed the study (48 weeks), FVC decreased by $\geqslant 10 \%$ in five $(29 \%)$ patients. Among the eight who discontinued, three were identified as decliners based on their performance in the period up to discontinuation. Thus, eight (32\%) out of 25 patients ( $95 \%$ CI 15-54\%) experienced a decline in lung function, while 17 (68\%) out of 25 were non-decliners. For the whole population, median FVC was $2.23 \mathrm{~L}$ (95\% CI $1.22-$ $3.80 \mathrm{~L})$ at study entry and $2.20 \mathrm{~L}(95 \%$ CI $0.99-4.71 \mathrm{~L})$ after 48 weeks. The percentage of decliners seen in our study was lower than the $54 \%(p=0.044)$ observed in the study by FLAHERTY et al. [7], the $55 \%$ observed in the placebo group in the etanercept trial (48 weeks) [8] and the 52\% reported in a Japanese pirfenidone trial (52 weeks) [9]. At least two measurements of FVC obtained before treatment were available in all patients. Application of a linear mixed model to assess the rate of decline of FVC before and after treatment over the entire cohort $(n=25)$ estimated the slopes to be $5.1 \%$ and $2.2 \%$ predicted per year $(p=0.76)$, respectively (data not shown). Similarly, a decrease in DL,CO of $\geqslant 10 \%$ was seen in $42 \%$ of patients (95\% CI $22-63 \%$ ), which is lower than the $69 \%$ observed in the study by FLAHERTY et al. [7]. Although our study is not strictly comparable with the other studies mentioned previously due to differences in sample size, characteristics of patient population, diagnostic criteria, therapeutic regimens, etc., these comparisons provide an overall perspective in which to evaluate our results.

A comparison of some of the primary and secondary end-points between decliners and non-decliners is given in table 1 . The most significant difference observed was for high-resolution CT lung alveolar score which tended to be lower in non-decliners $(p=0.053)$, i.e. patients who appeared to benefit from octreotide treatment. Clinical features, lung function tests, and levels of serum octreotide and fibrosis biomarkers were similar in the two groups. Among decliners, the percentage of nonsmokers was higher than among non-decliners (table 1). This may be attributed to the greater antifibrotic effects of octreotide in smokers, although there are no data to support this hypothesis. Alternatively, it may be due to smoking itself, which is a well-identified risk factor for IPF, although the links between tobacco smoking and decline in lung function remain a subject of debate [10]. A third possibility could be that emphysema, which is often associated with IPF in ex-smokers, compensates for the loss in FVC.

Interestingly, the lung uptake of ${ }^{111}$ In-octreotide measured at baseline before any octreotide administration tended to be higher in non-decliners $(p=0.11)$, suggesting that non-decliners had more octreotide receptors than decliners or that their rate of uptake per receptor was higher. This observation indirectly supports the notion of an antifibrotic action attributed to octreotide.

Patients included in this study appeared to be fairly stable over the study period (48 weeks) for all the recorded end-points: lung function tests, quality of life and blood biomarkers. Such stability of the disease was surprising, as the population was older than in most of the retrospective and prospective studies published to date, and had a rather severe disease grade based on a low DL,CO (32\% pred). 


\begin{tabular}{|c|c|c|c|}
\hline \multirow[t]{2}{*}{ TABLE 1} & \multirow[b]{2}{*}{ Non-decliners } & \multirow{2}{*}{\multicolumn{2}{|c|}{ p-value }} \\
\hline & & & \\
\hline \multicolumn{4}{|l|}{ Demographics } \\
\hline Subjects n & 17 & 8 & \\
\hline Age yrs & $69(53-78)$ & $72(63-80)$ & 0.32 \\
\hline Males/females n & $14 / 3$ & $4 / 4$ & 0.16 \\
\hline Never-smokers & $6(35)$ & $6(75)$ & 0.10 \\
\hline \multicolumn{4}{|l|}{ Lung function tests } \\
\hline FVC \% pred & $70(46-110)$ & $69(44-114)$ & 0.60 \\
\hline DL,CO \% pred & $35(17-92)$ & $29(24-44)$ & 0.33 \\
\hline Resting $\mathrm{Pa}, \mathrm{O}_{2} \mathrm{mmHg}$ & $77(53-91)$ & $76(61-101)$ & 0.77 \\
\hline \multicolumn{4}{|l|}{ HRCT scores } \\
\hline Alveolar score & $1.25(0.33-2.00)$ & $1.67(1.00-2.17)$ & 0.053 \\
\hline Interstitial score & $2.00(1.00-3.00)$ & $2.08(1.83-2.50)$ & 0.49 \\
\hline \multicolumn{4}{|l|}{ Biomarkers } \\
\hline SP-D ng $\cdot \mathrm{mL}^{-1}$ & 436 (110-1185) & $374(250-836)$ & 0.74 \\
\hline $\mathrm{KL}-6 \mathrm{U} \cdot \mathrm{mL}^{-1}$ & $1895(464-3857)$ & $1540(687-2832)$ & 0.61 \\
\hline $\mathrm{CCL} 2 \mathrm{pg} \cdot \mathrm{mL}^{-1}$ & 375 (152-1099) & 308 (144-1172) & 0.45 \\
\hline $\mathrm{CCL} 18 \mathrm{ng} \cdot \mathrm{mL}^{-1}$ & $138(64-395)$ & $118(61-275)$ & 0.74 \\
\hline Serum octreotide $\mathrm{ng} \cdot \mathrm{mL}^{-1}$ & $2.8(<0.5-6.04)$ & $2.6(<0.5-6.22)$ & 0.89 \\
\hline \multicolumn{4}{|l|}{$\begin{array}{l}\text { Uptake of octreotide in } \\
\text { the lungs }\end{array}$} \\
\hline${ }^{111}$ In-octreotide L/B & $5.32(3.90-8.87)$ & $4.36(3.49-5.81)$ & 0.11 \\
\hline \multicolumn{4}{|c|}{$\begin{array}{l}\text { Data are presented as mean (range) or } n(\%) \text {, unless otherwise stated. FVC: } \\
\text { forced vital capacity; \% pred: \% predicted; } \mathrm{DL}, \mathrm{CO} \text { : diffusing capacity of the lung } \\
\text { for carbon monoxide; } \mathrm{Pa}, \mathrm{O}_{2} \text { : arterial oxygen tension; HRCT: high-resolution } \\
\text { computed tomography; SP-D: surfactant protein-D; KL-6: Krebs von den } \\
\text { Lungen-6; CCL: CC chemokine ligand; L/B: lung/background ratio. }{ }^{*} \text { : patients } \\
\text { whose FVC decreased by } \geqslant 10 \% \text { between two consecutive measurements (12- } \\
\text { week interval) were termed as decliners and others as non-decliners. }\end{array}$} \\
\hline
\end{tabular}

Overall, the tolerability of octreotide was good and there were no deaths during the study. 85 adverse effects were reported in 20 patients. Adverse events were mainly gastrointestinal (diarrhoea) or respiratory (cough, increased dyspnoea and acute bronchitis) and generally mild. Seven severe adverse effects were reported, of which only one was thought to be related to octreotide (diarrhoea complicated with dehydration). One acute exacerbation of IPF was observed in a patient who had received 11 injections of octreotide.

In summary, this was a non-randomised, non-controlled study on 25 patients treated over 48 weeks. While the sample size and study design do not provide any definite conclusions, the results indicate that long-acting octreotide is well tolerated and provide a proof of concept that octreotide treatment slows down the progression of lung fibrosis. These results need to be confirmed by a larger placebo-controlled study.

\footnotetext{
B. Crestani ${ }^{*, \#, 9}$, J. Chapron", B. Wallaert ${ }^{+}$, E. Bergot ${ }^{\S}$,

P. Delaval ${ }^{f}$, D. Israel-Biet ${ }^{* *}$, J. Lacronique ${ }^{\# \#}$, I. Monnet $^{\text {ศथ }}$, M. Reynaud-Gaubert ${ }^{++}$, A. Tazi ${ }^{\S \S}$, R. Lebtahi ${ }^{f f}$, M-P. Debray ${ }^{* * *}$, M. Brauner ${ }^{\# \#}$, M. Dehoux ${ }^{* \uparrow \uparrow, ~ Q . ~ D o r n i c ~}{ }^{++, \S \S \S,}$ M. Aubier ${ }^{*, \#, q}$, F. Mentré ${ }^{+H, \S \S \S}$ and X. Duval ${ }^{\S \S \S, f f f}$
}

*Universite Paris Diderot, PRES Paris Cité, Inserm U700, ${ }^{\#}$ Service de Pneumologie A, ${ }^{f f}$ Service de Médecine Nucléaire, ***Service de Radiologie, "Taboratoire de Biochimie, ${ }^{++} \mathrm{UF}$ de Biostatistiques, ${ }^{f f f}$ Centre d'Investigation Clinique, Assistance Publique Hôpitaux de Paris, Hôpital Bichat, "Inserm, UMR 700, **Service de Pneumologie, Assistance Publique Hôpitaux de Paris, Hôpital Européen Georges Pompidou, \#\#service de Pneumologie, Assistance Publique Hôpitaux de Paris, Hôpital Cochin, ${ }^{\S \S}$ Service de Pneumologie, Assistance Publique Hôpitaux de Paris, Hôpital Saint Louis, ${ }^{\S \S \S I n s e r m, ~ U M R 738, ~ P a r i s, ~}{ }^{+} \mathrm{CHRU}$, Hôpital Calmette, Service des Maladies Respiratoires, Lille, ${ }^{\S} \mathrm{CHU}$ Côte de Nacre, Service de Pneumologie, Caen, ${ }^{\dagger}$ Service de Pneumologie, Hôpital Pontchaillou, Rennes, "Service de Pneumologie, Centre Hospitalier Intercommunal de Créteil, Créteil, ${ }^{+}$Service de Pneumologie, Assistance Publique Hôpitaux de Marseille, Hôpital de Sainte Marguerite, Marseille, and "\#\# Service de Radiologie, Assistance Publique Hôpitaux de Paris, Hôpital Avicenne, Bobigny, France.

Correspondence: B. Crestani, Service de Pneumologie A, Centre de Compétence des Maladies Pulmonaires Rares, Hôpital Bichat, 46 rue Henri Huchard, 75877 Paris Cedex 18, France. E-mail: bruno.crestani@bch.aphp.fr

Support Statement: Octreotide was kindly donated by Novartis Pharma S.A.S. (Rueil-Malmaison, France).

Clinical Trial: This study is registered at Clinicaltrials.gov with identifier number NCT00463983.

Statement of Interest: A statement of interest for this study can be found at www.erj.ersjournals.com/site/misc/statements.xhtml

Acknowledgements: The authors acknowledge the support of S. Bisot-Locard (Novartis Pharma S.A.S, Rueil-Malmaison, France), H. Papayan and C. Vergne as clinical research assistants, J. Benessiano and the Centre for Biological Resources, and A. Certain (all Bichat Hospital, Paris, France) for help in the drug management of the trial.

\section{REFERENCES}

1 Noble PW, Albera C, Bradford WZ, et al. Pirfenidone in patients with idiopathic pulmonary fibrosis (CAPACITY): two randomised trials. Lancet 2011; 377: 1760-1769.

2 Borie R, Fabre A, Prost F, et al. Activation of somatostatin receptors attenuates pulmonary fibrosis. Thorax 2008; 63: 251-258.

3 Lebtahi R, Moreau S, Marchand-Adam S, et al. Increased uptake of ${ }^{111}$ In-octreotide in idiopathic pulmonary fibrosis. J Nucl Med 2006; 47: 1281-1287.

4 American Thoracic Society. Idiopathic pulmonary fibrosis: diagnosis and treatment. International consensus statement. American Thoracic Society (ATS), and the European Respiratory Society (ERS). Am J Respir Crit Care Med 2000; 161: 646-664.

5 Ley B, Collard HR, King TE Jr. Clinical course and prediction of survival in idiopathic pulmonary fibrosis. Am J Respir Crit Care Med 2011; 183: 431-440.

6 van den Blink B, Wijsenbeek MS, Hoogsteden HC. Serum biomarkers in idiopathic pulmonary fibrosis. Pulm Pharmacol Ther 2010; 23: 515-520. 
7 Flaherty KR, Mumford JA, Murray S, et al. Prognostic implications of physiologic and radiographic changes in idiopathic interstitial pneumonia. Am J Respir Crit Care Med 2003; 168: 543-548.

8 Raghu G, Brown KK, Costabel U, et al. Treatment of idiopathic pulmonary fibrosis with etanercept: an exploratory, placebo-controlled trial. Am J Respir Crit Care Med 2008; 178: 948-955.
9 Taniguchi H, Ebina M, Kondoh Y, et al. Pirfenidone in idiopathic pulmonary fibrosis. Eur Respir J 2010; 35: 821-829.

10 Antoniou KM, Hansell DM, Rubens MB, et al. Idiopathic pulmonary fibrosis: outcome in relation to smoking status. Am J Respir Crit Care Med 2008; 177: 190-194.

\section{Obstructive lung function in sarcoidosis may be missed, especially in older white patients}

\section{To the Editors:}

Lung function abnormalities are often present when patients are first diagnosed with pulmonary sarcoidosis, and most studies to date indicate that the predominant pattern is of restriction or normal airways, with a truly obstructive pattern in a minority [1]. The largest case-control study of sarcoidosis to date, the ACCESS (A Case Control Etiologic Study of Sarcoidosis) trial, showed that the biggest group of patients in that particular cohort $(46.9 \%)$ presented with a forced expiratory volume in $1 \mathrm{~s}$ (FEV1)/forced vital capacity (FVC) ratio of $>80 \%$ and only $13.2 \%$ presented with a ratio of $50-69 \%$ [2]. Of the 215 patients from the ACCESS trial who were followed up for a further 2 yrs, the majority showed no change in pulmonary function, radiographic stage or dyspnoea score [3]. A more recent European study showed that patients with sarcoidosis had impaired lung compliance and a reduced diffusing capacity of the lung for carbon monoxide (DL,CO) and, while there was no predominance of restrictive lung defects, airway obstruction was only present in $11.7 \%$ of patients [4].

Over the past 3 yrs, we have noticed that an increasing number of patients who present to our clinics in West London, UK with a new diagnosis of pulmonary sarcoidosis have a predominantly obstructive lung defect. A detailed analysis of lung function in sarcoidosis with respect to stratification by patient demographics has, to our knowledge, not been previously reported. We present the results of a systematic retrospective analysis of 164 consecutive patients presenting with a new diagnosis of pulmonary sarcoidosis over a 10-yr period and in whom formal lung function was recorded at the time of presentation. All patients had a clinically definite diagnosis, with supportive histology where available, and the subsequent follow-up examinations were in keeping with the original diagnosis. Lung function testing was carried out using a standardised protocol (single-breath method for gas transfer and nitrogen washout for lung volumes) and recorded electronically. All testing was performed at a single institution and statistical comparisons were performed by MannWhitney U-tests and multiple linear regression analysis.

Our data show that the percentage of patients presenting with an obstructive lung defect of FEV1/FVC ratio 50-69\% was higher than expected, at $23.2 \%$ (fig. 1a), while $42.1 \%$ of patients presented with FEV1/FVC $>80 \%$. There was no significant difference in smoking status (the majority were nonsmokers), diagnosis of asthma or chronic obstructive pulmonary disease (COPD) between the obstructive and restrictive groups. We also found that patients with an obstructive defect (median $53.5 \mathrm{yrs}$, interquartile range (IQR) 39-61.25 yrs) were significantly older $(p=0.0015)$ than those with restrictive defects (median 44 yrs, IQR 33-50.5 yrs) (fig. 1b).

A significant difference $(p=0.028)$ was found in lung function between the main ethnic groups, with black patients having a higher FEV1/FVC ratio (median 81\%, IQR 73-85\%) than white patients (median 78\%, IQR 69-83\%). In addition, the proportions of lung function pattern within ethnicities was dramatically different (fig. 1c). White patients $(n=83)$ presented with a restrictive/obstructive ratio of 1.4 , while this ratio was more than twice as large in black patients $(n=54)$, at 4.0. The ratio of Asian (Indian subcontinent) patients $(n=27)$ was similar to that in the white population, at 1.3. There was no difference found between the ethnicities in terms of other measured lung function parameters, including the $\mathrm{DL}, \mathrm{CO} \%$ predicted (fig. 1d), residual volume or FVC.

On multivariate analysis of the black patient group (online supplementary table 1), there were no significant demographic or clinical associations found, suggesting that the greater proportion of black patients seen presenting with a restrictive lung pattern in this cohort was not due to factors such as presence of co-existing lung disease or demographic differences (e.g. 5.6\% of black patients and $6 \%$ of white patients were recorded as having asthma or COPD at the time of diagnosis of sarcoidosis). Only a small number of patients with obstructive lung defects were assessed for bronchodilator response, but in those who were $(n=9)$, we saw no significant improvement in lung function (mean change in FEV1 5\%, range -8-13\%).

Almost one in four of all patients in this cohort presented with an FEV1/FVC ratio of $50-69 \%$ and this obstructive pattern of lung function was significantly more common in those who were older and who were white. One possible explanation for our findings of a larger than expected group with significant airway obstruction may be our routine use of endobronchial ultrasound sampling for lymph node disease. This relatively specialised method, with greater diagnostic sensitivity in sarcoidosis [5], may be providing a histological confirmation in patients in whom a diagnosis of 